\section{Case Reports in Oncology}

\title{
Primary Pleural Hemangioendothelioma: A Case Report and Literature Review
}

\author{
Elham Askari ${ }^{a}$ Shekoofeh Yaghmaei ${ }^{b} \quad$ Sara Haselic \\ Mihan Pouradollah Totkaboni ${ }^{a}$ \\ aDepartment of Pathology, Chronic Respiratory Diseases Research Center, National \\ Research Institute of Tuberculosis and Lung Diseases (NRITLD), Shahid Beheshti University \\ of Medical Sciences, Tehran, Iran; bepartment of General Medicine, Shiraz University of \\ Medical Sciences, Shiraz, Iran; 'Department of Radiology, Chronic Respiratory Diseases \\ Research Center, National Research Institute of Tuberculosis and Lung Diseases (NRITLD), \\ Shahid Beheshti University of Medical Sciences, Tehran, Iran
}

\section{Keywords}

Pleural hemangioendothelioma $\cdot$ Epithelioid hemangioendothelioma $\cdot$ Computed tomography

\begin{abstract}
Epithelioid hemangioendothelioma (EHE) is a rare tumor of the vascular endothelial cells. It can originate from any tissue; however, it occurs most frequently in the liver and lung. Pleural epithelioid hemangioendothelioma (PEH), EHE developing from pleura, is even more infrequent and less reported in the literature. In the following report, we discuss a 40 -year-old man who initially presented with right-sided chest pain. Computed tomography revealed pleural thickening and effusion in his right thoracic cavity. PEH diagnosis was confirmed with immunohistochemistry. In order to provide the readers with an inclusive understanding of the disease, we collected the PEH cases reported in the literature. Despite the scarcity of the reported PEH cases (to our best knowledge), the compiled literature review of the disease enables the readers to grasp a better comprehension of the disease.
\end{abstract}

(C) 2021 The Author(s).

Published by S. Karger AG, Basel

\section{Introduction}

Epithelioid hemangioendothelioma (EHE) is a rare intermediate malignant tumor originating from vascular endothelial cells. EHE can be located in variable organs, including the lung, liver, bone, soft tissue, skin, gastrointestinal tract, brain, mediastinum, spleen, breast, 
Case Reports in Oncology
Case Rep Oncol 2021;14:1201-1211

\begin{tabular}{l|l}
\hline DOI: 10.1159/000518243 & ๔ 2021 The Author(s). Published by S. Karger AG, Basel
\end{tabular} www.karger.com/cro

Askari et al.: Primary Pleural Hemangioendothelioma

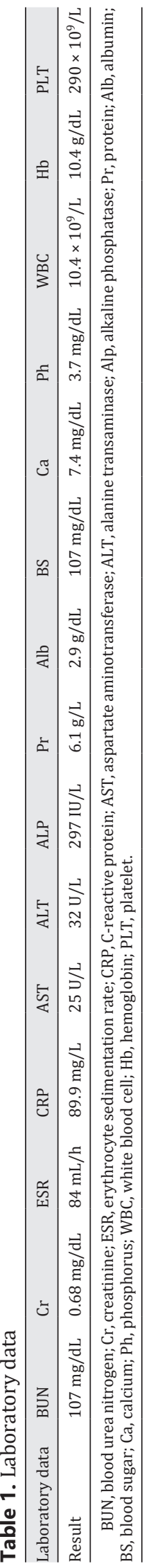

Karger' 

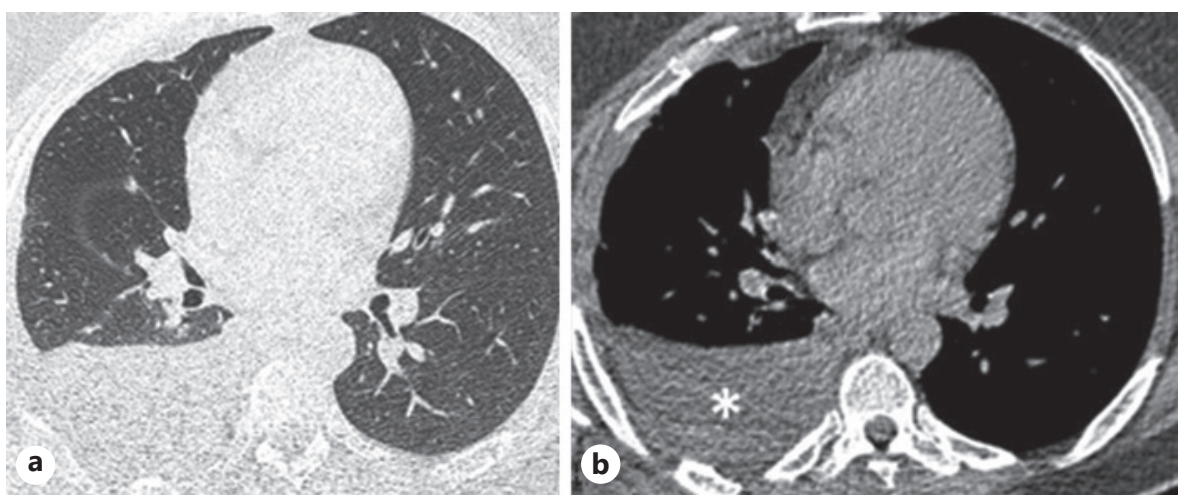

Fig. 1. a, b Axial noncontrast CT scan revealed right-sided pleural effusion without underlying parenchyma lesion. CT, computed tomography.

testis, thyroid, and heart [1]. EHE patients develop metastatic disease in 20-30\% of cases. The etiology of EHE is not yet well-understood. Despite being less frequent, primary pleural hemangioendothelioma (PEH) is believed to be more aggressive than other EHE subtypes [2]. The 5-year EHE overall survival rate is $>70 \%$ [3], whereas it is much less optimistic in PEH [4].

Here, we describe a 40-year-old male patient presented with right-sided chest pain diagnosed with PEH. Additionally, we discussed the relevant literature in order to advance the understanding of the symptoms and diagnosis of the disease.

\section{Case Report}

In July 2020, a 40-year-old man was referred to our center, a university-affiliated respiratory hospital, complaining of right-sided chest pain accompanied by dyspnea for 1 year. He began experiencing symptoms while noticing a sharp and pleuritic pain in his right-sided chest radiating to the right neck. Besides these, he did not mention any other symptoms, such as fever or weight loss. His previous medical history was noncontributory, and he denied a history of smoking or asbestos exposure. Aside from that, he has not ever received radiotherapy. At the time of admission, our patient was hemodynamically stable, and the physical examination was unremarkable. Hematologic laboratory and blood chemical test results are demonstrated in Table 1.

He underwent an initial chest radiograph which revealed right-sided pleural effusion. Consequently, a computed tomography (CT) scan was requested, which demonstrated rightsided pleural effusion and thickening without underlying parenchymal abnormality (Fig. 1a, b). Also, the adjacent lung passively collapsed.

Echocardiography established proper cardiac function and (ejection fraction $=63 \%$ ) without any structural abnormality. Also, there was no sign of pericardial effusion.

Afterward, a thoracocentesis was done. The pleural fluid analysis is given in detail in Table 2. Cytological examination of the pleural fluid was unremarkable.

To further assess the patient, a video-assisted thoracoscopic surgery (VATS) was requested, in which the following were observed: right-sided pleural thickening, visceral pleural nodularity, and massive effusion. A pleural biopsy was obtained during VATS, the results of which are as follows: microscopically, the pleural tissue composed of single cells, cords, and nests of atypical polygonal cells with round, reniform nuclei, some vesicular chromatin, and abundant pale eosinophilic vacuolated cytoplasm. Intracytoplasmic vacuoles occasionally contain an erythrocyte. In some foci, the atypical cells were surrounded by the inflammatory cells. Also, no increased mitotic activity, necrosis, or prominent pleomorphism was demonstrated (Fig. 2a).

\section{Karger'}


Case Reports in Oncology
Case Rep Oncol 2021;14:1201-1211

\begin{tabular}{l|l}
\hline DOI: $10.1159 / 000518243$ & (c) 2021 The Author(s). Published by S. Karger AG, Basel
\end{tabular} www.karger.com/cro

Askari et al.: Primary Pleural Hemangioendothelioma

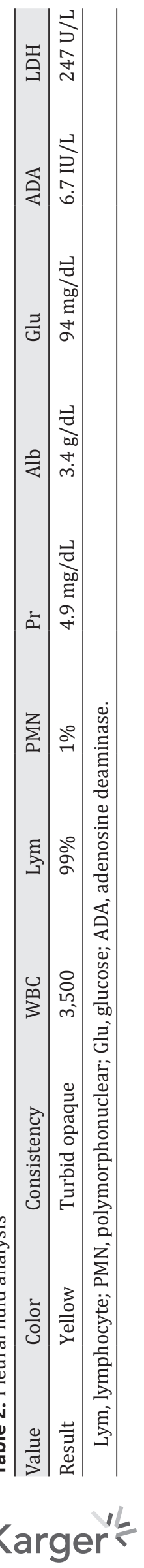



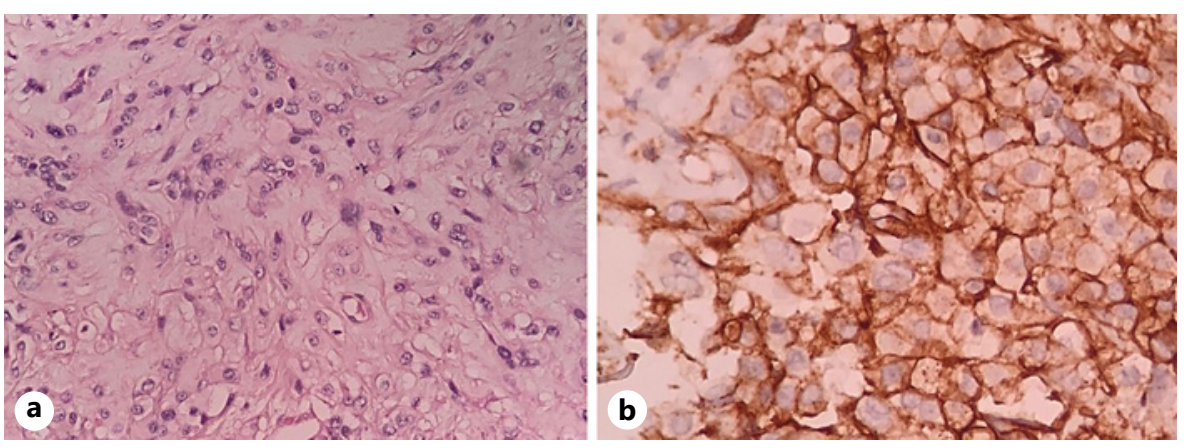

Fig. 2. Pleural biopsy specimen. a Hematoxylin and eosin staining exhibited a nest of atypical epithelioid cells with vacuolated cytoplasm. b Membranous CD31 immunostaining.

During immunohistochemistry staining, the mentioned cells showed high levels of vimentin, CD31, and CD10. A group of cells was found to react positively to CD34 and EMA, while no reaction was seen to WT1, and calretinin was negative (Fig. 2b). In addition, the proliferation index by Ki67 was up to $15 \%$. As a result of the histopathological findings, the patient was diagnosed with PEH.

There were no findings of metastasis on CT scans of the abdomen and pelvis. A bone scan exhibited increased uptake in the right hemithorax, representing the possibility of malignancy or simply due to the previous manipulation. Also, the radiopharmaceutical agent appeared to be symmetrically taken up in both tibial cortexes, suggesting hypertrophic pulmonary osteoarthropathy (Fig. 3a, b).

Eventually, the patient was referred to oncology, where he was started on a regimen of Adriamycin and ifosfamide. The patient is currently undergoing chemotherapy and has not mentioned particular problems.

\section{Literature Review}

We reviewed the related literature published since 1987 (a total of 22 cases) in Table 3. According to these reports and our own, we have discussed the characteristics of this disease.

\section{Discussion}

EHE is a rare vascular tumor. From the clinical perspective, the tumor behavior is between benign hemangiomas and high-grade angiosarcomas [22]. Even though EHE can arise in different body parts, it has a higher probability of developing in the liver and lung. Female individuals are generally affected by EHE more than male counterparts. The peak incidence of the disease is in the fourth and the fifth decade of life.

In contrast, PEH typically occurs more frequently in older male individuals [2]. In our analysis of the 23 cases identified in the literature, $52 \%$ of patients were male, with a mean age of 50 years. Dyspnea is shown to be the most prevalent symptom of PEH, followed by chest pain and cough, which our study was well-aligned with (overall data indicated that the most common presented symptom was dyspnea reported by $60 \%$ of patients). Also, a group of patients complained about back pain, weight loss, fatigue, and anorexia. Since some patients might be asymptomatic at diagnosis or be experiencing minor symptoms, $\mathrm{PEH}$ is discovered in some cases through incidental diagnosis.

\section{Karger'}




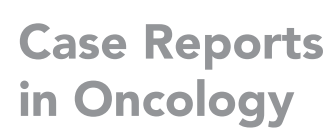

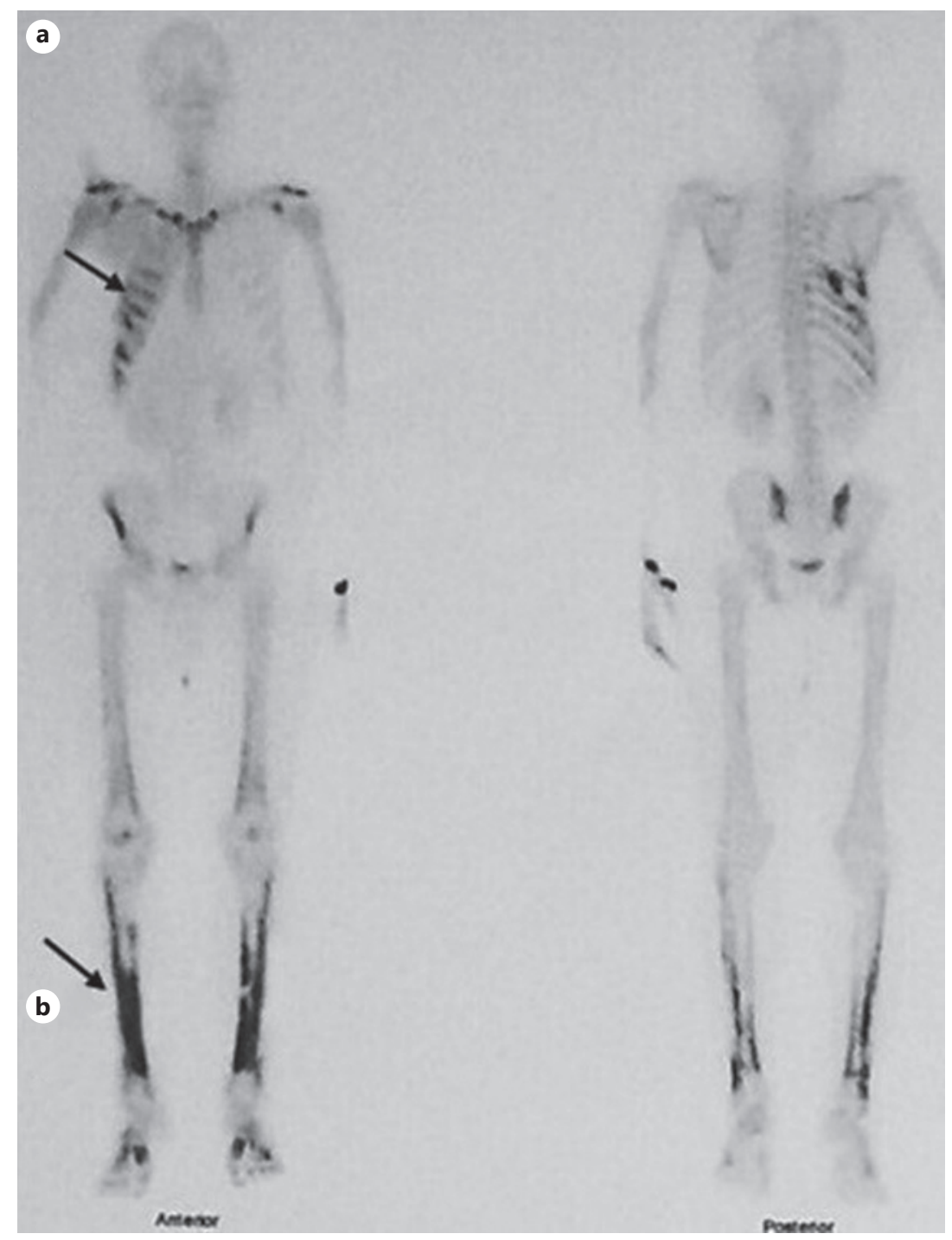

Fig. 3. Bone scan showed increased radiopharmaceutical uptake: in right hemithorax (postoperative changes) (a), along both tibial cortexes suggesting HPO (b). HPO, hypertrophic pulmonary osteoarthropathy.

Despite the lack of correlation between smoking and PEH, radiation and asbestos exposure are considered related factors. Moreover, 5CAMTA1 gene rearrangements; translocations in chromosomes 7, 14, and 22; the loss of Y chromosome; and t (1; 3) (p36; q23-25) [20] have been reported as predisposing factors for PEH. It is worth mentioning that the majority of hematological and laboratory tests are not specific.

Slight (or moderate) unilateral pleural effusion is the most common chest CT finding in PEH patients, frequently accompanied by pleural thickening. For the case reported here, chest CT showed right thoracic collapse and diffuse pleural thickening. The changes were quite similar to those reported in the other literature. Pleural effusion is more common on the right side (20). (In our review, 57\% of PEH were diagnosed on the right side.)

PEH consists of relatively monomorphic epithelioid cells arranged in cords and nests within a myxohyaline stroma from the pathological perspective. Cell nuclei are eccentric, vacuolated, and similar to signet ring cells. The cytoplasm is eosinophilic, with vacuoles of varying sizes in the cytoplasm of some tumor cells. It is also notable that immunohistochemical 


\section{Case Reports in Oncology}

Case Rep Oncol 2021;14:1201-1211

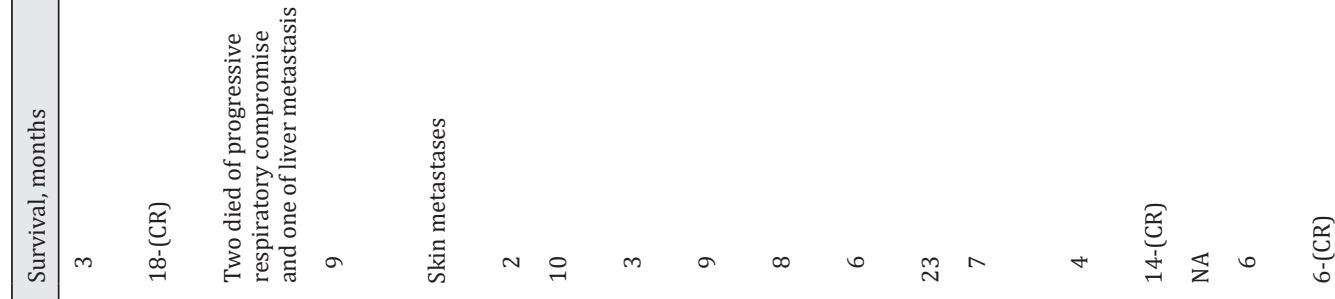

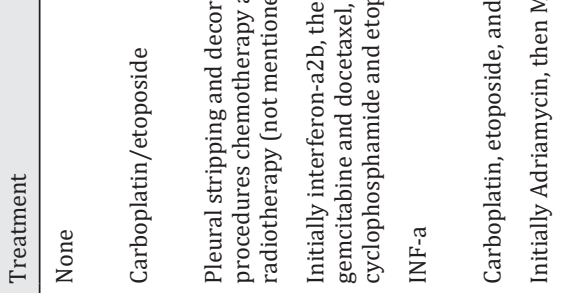

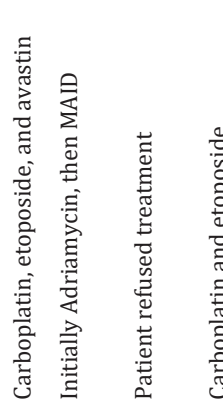

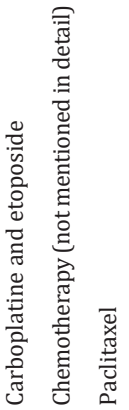
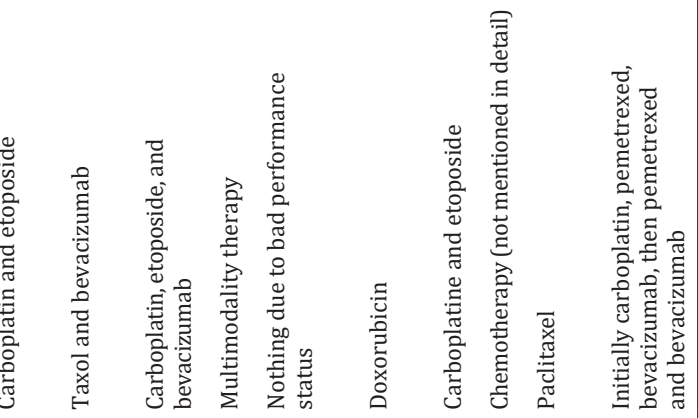
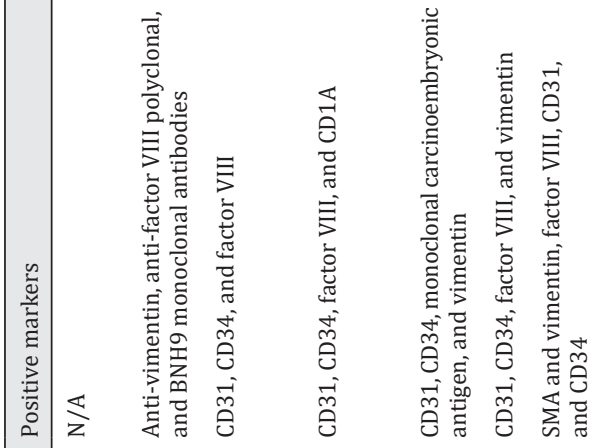

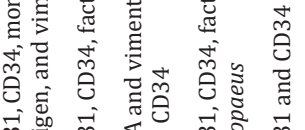

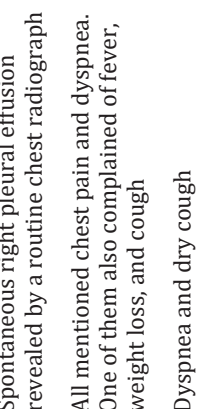
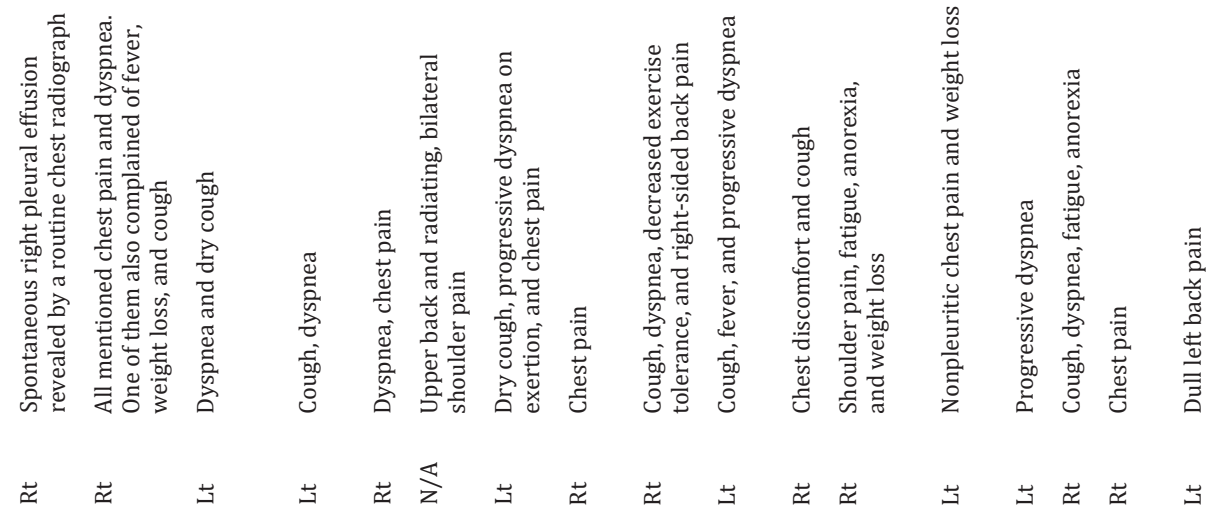
Case Reports in Oncology
Case Rep Oncol 2021;14:1201-1211

\begin{tabular}{l|l}
\hline DOI: $10.1159 / 000518243$ & (c) 2021 The Author(s). Published by S. Karger AG, Basel
\end{tabular} www.karger.com/cro

Askari et al.: Primary Pleural Hemangioendothelioma

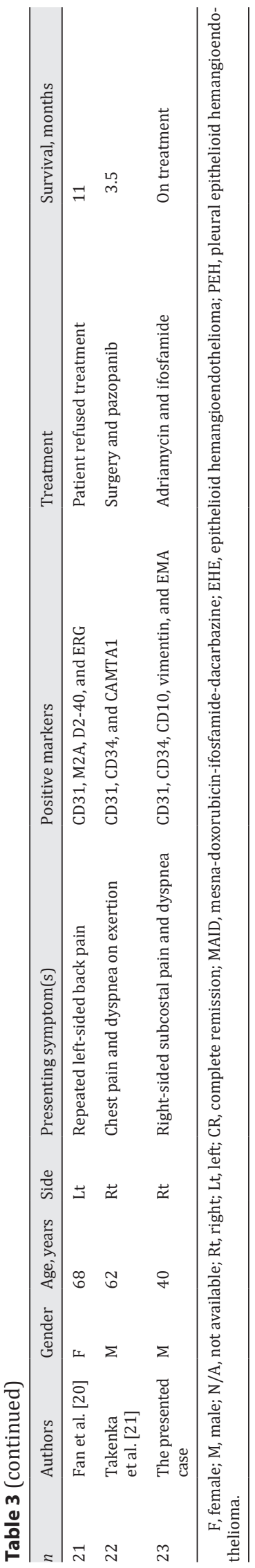

Karger ${ }^{\prime \prime}$ 
staining is the most reliable diagnostic means to determine the origin of tumor cells. Vascular endothelial cell markers can be detected with this staining method; among them, CD31 and CD34 exhibit the highest specificity [23]. According to our literature review, CD31, CD34, and factor VIII were the most prevalent positive markers. In the case presented earlier, the immunohistochemical staining result was positive for CD31 and CD34 as well.

EHE tumors are radio- and chemoresistant [23]. Radiotherapy is reserved for the palliative therapies of cases with bone involvement, and there have not been homogeneous responses obtained from chemotherapy schemes. PEH is often presented at an advanced stage and can rarely be resected for cure [2]. Therefore, most of the patients undergo chemotherapy mainly with carboplatin and etoposide [12]. Different results from both surgical treatment and chemotherapy have been reported in different studies (showed in Table 3); however, reports are pretty conflicting, and many studies have described cases in which combination chemotherapy of carboplatin and etoposide was ineffective [12].

Metastases, mesotheliomas, sarcomas (such as angiosarcoma or Kaposi), hyperplasia, tuberculosis, and pseudopyogenic granuloma are PEH differential diagnoses [14], which physicians should consider. Despite the localized infiltration in the early stages, pleura involvement becomes more extensive as the disease progresses. As a result, neither pleuroscopy nor VATS is a reliable technique to diagnose PEH in the early stages of the disease. Even if the aforementioned methods are not diagnostic initially, it is crucial to keep an eye on suspicious cases.

\section{Conclusion}

The patient presented before and similar reported cases may improve the clinical understanding of PEH. Since the clinical manifestation of PEH is not specific, it is often misdiagnosed in the early stages. Consequently, we highly recommend considering PEH as a differential diagnosis for patients with pleural thickening.

\section{Acknowledgements}

We thank Ali Falsafi for his valuable help in editing this manuscript.

\section{Statement of Ethics}

Written informed consent has been received from the patient. It is in the Persian language (the mother tongue of the patient). We will send it to the respectful journal if needed. The study protocol was approved by the Shahid Beheshti University of Medical Sciences Ethics Committee. The reference number is IR. SBMU.NRITLD.REC.1400.011.

\section{Conflict of Interest Statement}

None.

\section{Funding Sources}

None.

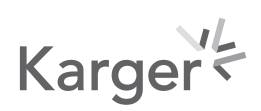




\section{Case Reports in Oncology}

Case Rep Oncol 2021;14:1201-1211

\begin{tabular}{l|l}
\hline DOI: $10.1159 / 000518243$ & (c) 2021 The Author(s). Published by S. Karger AG, Basel
\end{tabular} www.karger.com/cro

\section{Author Contributions}

Elham Askari: she was the pathologist who presented the case and made the patient's diagnosis and handed in the sample's figures. Shekoofeh Yaghmaei: original draft preparation and reviewing the manuscript. Sara Haseli: she was the radiologist who did the chest CT and reported it. Mihan Pouradollah Totkaboni: she is the pathologist who approved the diagnosis on sample immunostaining. All authors read and evaluated the final manuscript.

\section{Data Availability Statement}

All data analyzed during this study are included in this article. Further inquiries can be directed to the corresponding author.

\section{References}

1 Salech F, Valderrama S, Nervi B, Rodriguez JC, Oksenberg D, Koch A, et al. Thalidomide for the treatment of metastatic hepatic epithelioid hemangioendothelioma: a case report with a long term follow-up. Ann Hepatol. 2016;10(1):99-102.

2 Lee YJ, Chung MJ, Jeong KC, Hahn CH, Hong KP, Kim YJ, et al. Pleural epithelioid hemangioendothelioma. Yonsei Med J. 2008;49(6):1036.

3 Deyrup AT, Tighiouart M, Montag AG, Weiss SW. Epithelioid hemangioendothelioma of soft tissue: a proposal for risk stratification based on 49 cases. Am J Surg Pathol. 2008;32(6):924-7.

4 Crotty EJ, McAdams HP, Erasmus JJ, Sporn TA, Roggli VL. Epithelioid hemangioendothelioma of the pleura: clinical and radiologic features. AJR Am J Roentgenol. 2000;175(6):1545-9.

5 Yousem SA, Hochholzer L. Unusual thoracic manifestations of epithelioid hemangioendothelioma. Arch Pathol Lab Med. 1987;111(5):459-63.

6 Pinet C, Magnan A, Garbe L, Payan MJ, Vervloet D. Aggressive form of pleural epithelioid haemangioendothelioma: complete response after chemotherapy. Eur Respir J. 1999;14(1):237-8.

7 Cronin P, Arenberg D. Pulmonary epithelioid hemangioendothelioma: an unusual case and a review of the literature. Chest. 2004;125(2):789-93.

8 Al-Shraim M, Mahboub B, Neligan PC, Chamberlain D, Ghazarian D. Primary pleural epithelioid haemangioendothelioma with metastases to the skin. A case report and literature review. J Clin Pathol. 2005; 58(1):107-9.

9 Saqi A, Nisbet L, Gagneja P, Leslie KO. Primary pleural epithelioid hemangioendothelioma with rhabdoid phenotype: report and review of the literature. Diagn Cytopathol. 2007;35(4):203-8.

10 Bocchino M, Barra E, Lassandro F, Ranieri F, Muto R, Rea G. Primary pleural haemangioendothelioma in an Italian female patient: a case report and review of the literature. Monaldi Arch Chest Dis. 2010;73(3):135.

11 André ST, Valente C, Paiva B, Pêgo A, Carvalho L, Luís AS. [Epithelioid hemangioendothelioma of the pleura: a rare presentation of a clinical case]. Rev Port Pneumol. 2010;16(3):477-82.

12 Lazarus A, Fuhrer G, Malekiani C, McKay S, Thurber J. Primary pleural epithelioid hemangioendothelioma (EHE)-two cases and review of the literature. Clin Respir J. 2011;5(1):e1-5.

13 Kim EA, Lele SM, Lackner RP. Primary pleural epithelioid hemangioendothelioma. Ann Thorac Surg. 2011; 91(1):301-2.

14 Márquez-Medina D, Samamé-Pérezvargas JC, Tuset-DerAbrain N, Montero-Fernández A, Taberner-Bonastre T, Porcel JM. Pleural epithelioid hemangioendothelioma in an elderly patient. A case report and review of the literature. Lung Cancer. 2011;73(1):116-9.

15 Bansal A, Chawla M, Cohen PJ, Kwon JS. Pleural epithelioid hemangioendothelioma. Lung. 2012;190(4):469.

$16 \mathrm{Yu} \mathrm{L}, \mathrm{Gu}$ T, Xiu Z, Shi E, Zhao X. Primary pleural epithelioid hemangioendothelioma compressing the myocardium. J Card Surg. 2013;28(3):266-8.

17 Ha SY, Choi IH, Han J, Choi YL, Cho JH, Lee KJ, et al. Pleural epithelioid hemangioendothelioma harboring CAMTA1 rearrangement. Lung Cancer. 2014;83(3):411-5.

18 Salijevska J, Watson R, Clifford A, Ritchie AI, Mauri F, Adeboyeku D. Pleural epithelioid hemangioendothelioma: literature summary and novel case report. J Clin Med Res. 2015;7(7):566.

19 Kanemura S, Kuribayashi K, Moriya Y, Shimizu S, Tsujimura T, Nakano T. Pemetrexed for epithelioid haemangioendothelioma of the pleura. Respirol Case Rep. 2016;4(6):e00191.

20 Fan Y, Wang F, Li S, Ye C, Ying Y, Mao H. Pleural epithelioid hemangioendothelioma: a case report and literature review. J Natl Med Assoc. 2016;108(2):124-9.

21 Takenaka M, Ichiki Y, Nabe Y, Tsuda Y, Kuwata T, Chikaishi Y, et al. Difficulty of treatment for pleural epithelioid hemangioendothelioma: a report of a case. Gen Thorac Cardiovasc Surg. 2020;68(2):190-3. 


\section{Case Reports in Oncology}

22 Rosenbaum E, Jadeja B, Xu B, Zhang L, Agaram NP, Travis W, et al. Prognostic stratification of clinical and molecular epithelioid hemangioendothelioma subsets. Mod Pathol. 2020;33(4):591-602.

23 Bahrami A, Allen TC, Cagle PT. Pulmonary epithelioid hemangioendothelioma mimicking mesothelioma. Pathol Int. 2008;58(11):730-4. 